\title{
Modelling feedlots using the MEDLI model framework
}

\author{
$\underline{\text { A.M. Vieritz }}^{\mathrm{a}}$, M.M. Lowry ${ }^{\mathrm{b}}$, T.J. Sullivan ${ }^{\mathrm{b}}$, M.G. Atzeni ${ }_{2}^{\mathrm{c}}$ A.J. Hope-O'Connor ${ }^{\mathrm{a}}$ and P.J. Watts ${ }^{\mathrm{b}}$ \\ ${ }^{a}$ Department of Environment and Science, QLD, ${ }^{b}$ Premise Pty. Ltd., QLD, ${ }^{c}$ Department of Agriculture and \\ Fisheries, QLD. \\ Email: Alison.Vieritz@des.qld.gov.au
}

\begin{abstract}
Model for Effluent Disposal using Land Irrigation (MEDLI) is a biophysically-based daily timestep model released in 1996 to facilitate designing effluent irrigation schemes. The model simulates a waste stream generator producing effluent that is treated in a pond system with a wet weather storage pond from which the effluent is irrigated as required to an area of land growing vegetation (Gardner et al. 1996). To complement the existing waste stream generator options, MEDLI is undergoing further development to include rainfall-dependent waste streams, including that generated by rainfall wash-off from feedlot production pens. This will facilitate MEDLI's use for designing effluent irrigation schemes associated with feedlots.

The feedlot pen model attempts to model the complex dynamic processes within feedlot production pens that impact on the quantity and quality of runoff using a daily time-step mass balance approach. An early description of the feedlot model for MEDLI, focusing on runoff quantity, was provided by Atzeni et al. (2001). Since then, the hydrology component has been substantially improved to generate daily surface and sub-surface pad moisture output for use in predicting odour emissions (Atzeni et al. 2015), as well as runoff quantity and quality. In this paper, we present the modelling approach and model algorithms used to simulate the waste stream from the feedlot production pens. Supporting references are detailed in Atzeni et al. (2015).

The MEDLI feedlot pen model is designed to simulate a modern feedlot yard with equal-sized production pens having adequate slope, and operating within the recommended Australian guidelines. Cattle can be designated to up to four markets, with market-specific entry and exit weights, daily weight gain, proportion of total herd designated, and proportion of pens occupied. Daily calculations are performed on a pen by pen basis, to model the key processes of herd dynamics, manure (faeces+urine) production, assimilation of the fresh manure into the pad, pen hydrology and pen cleaning. Herd dynamics include modelling animal mortality and pen stocking. When animals in a pen reach the exit weight for their market type, the model flags that the pen is vacant and drafts another mob (of the same market type) into another vacant pen if possible, or else the same pen. Manure production relies on BEEFBAL (QPIF 2004) or similar model to provide the market-specific annual manure production (total solids, volatile solids, total nitrogen, total phosphorus, salts and water) of each animal which is then used to determine the solids, nutrient, salt and water loading onto the manure pad. Assimilation of the fresh manure into the pad uses a two-layer model for the manure pad, assuming no loss of water or solids below the lower layer of the pad. The two layers capture the dynamics of pad hydrology and composition, including the impacts of rainfall, evaporation, animal stocking, manure accumulation, volatile solids decay, pen cleaning, runoff and manure erosion during runoff. Pens are cleaned at intervals to remove the excess manure, and involve considering the specified minimum number of days since a pen is cleaned, the pen's pad moisture content, pad depth, and the number of pens being cleaned each day. By modelling these processes, the fate of the nutrients, salts and solids from the manure pads is simulated as shown in Figure 1.
\end{abstract}

Validation of the feedlot pen model hydrology was undertaken using four field-collected data sets from three South East Queensland feedlots. The prediction of runoff quantity appears closely correlated with measured data. However, the runoff quality predictions require calibration of the total nitrogen, total phosphorus, and salt runoff concentrations with actual or expected holding pond chemistry. Data collection is in progress to allow further testing and validation of the feedlot pen module.

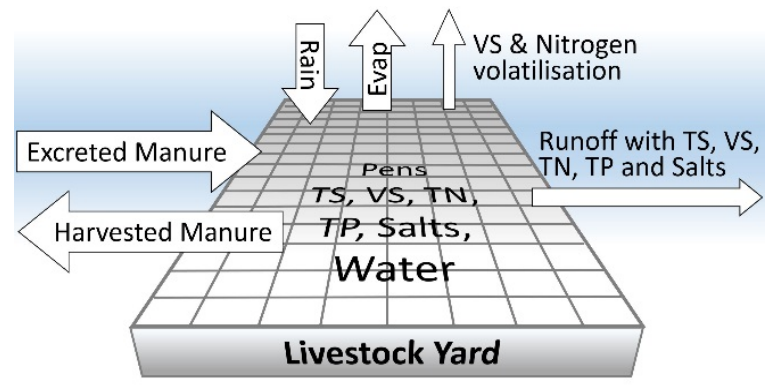

Figure 1. Key processes modelled to describe the movement of total solids (TS), volatile solids (VS), total nitrogen (TN), total phosphorus (TP), salts and water in feedlot production pens.

Keywords: MEDLI, model for effluent disposal using land irrigation, feedlot pad runoff modelling 


\section{INTRODUCTION}

Model for Effluent Disposal using Land Irrigation (MEDLI) is a biophysically-based daily time-step model released in 1996 to facilitate designing effluent irrigation schemes. The model simulates a waste stream generator producing effluent that is treated in a pond system with a wet weather storage pond from which the effluent is irrigated as required to an area of land growing vegetation (Gardner et al. 1996). To complement the existing waste stream options, MEDLI is undergoing further development to include rainfall-dependent waste streams, including that generated by rainfall wash-off from feedlot production pens. This will facilitate MEDLI's use for designing effluent irrigation schemes associated with feedlots.

The feedlot pen model attempts to model the complex dynamic processes within feedlot production pens that impact on the quantity and quality of runoff using a daily time step mass balance approach. An early description of the feedlot model (Atzeni et al. 2001) focused on runoff quantity. Since then, the hydrology component has been substantially improved to generate daily surface and sub-surface pad moisture output for use in predicting odour emissions (Atzeni et al. 2015), as well as runoff quantity and quality. In implementing the feedlot pen model into the MEDLI V2 framework, we have adopted a flexible approach so that the user can define multiple waste streams for a single scenario (not previously possible) and hence model a feedlot enterprise within a single model scenario by simulating rainfall-dependent (runoff) waste streams from feedlot production pens, vegetated surfaces, non-vegetated surfaces and roofs. These additional waste streams complement the existing sewage treatment plant and generic waste stream generator options. In this paper, we present the model algorithms used to simulate the waste stream from the feedlot production pens only. Supporting references are detailed in Atzeni et al. (2015).

\section{MODEL DESCRIPTION}

The MEDLI feedlot pen model simulates a modern feedlot yard with equal-sized production pens having adequate slope, and operating within the recommended Australian guidelines. Excreted manure (urine + faeces) forms into a manure pad with an impermeable organic layer or interface above the base material (typically well-compacted clay or gravel) of the pen. The manure pad is assumed to be uniform in depth, and homogenous with respect to nutrient and salt composition but non-homogenous (two layers) with respect total solids (TS), volatile solids (VS) and water. The fate of total nitrogen (TN), total phosphorus (TP), salts, and solids from the manure pad is simulated as shown in Figure 1. To simulate these dynamic processes, user defined inputs are required. All user-specified inputs with their units except climate inputs are shown in Figure 2 and appear in the italicised algorithm descriptions below concatenated and highlighted in bold. A source file is specified by the user for daily Rain ( $\mathrm{mm}$ ), PanEvaporation $(\mathrm{mm})$ and AverageAirTemperature $\left({ }^{\circ} \mathrm{C}\right)$ data.

\subsection{Feedlot pen initialisation}

From the Livestock Yard Characteristics inputs (Figure 2), the production pen area can be calculated (1). The total production pen area does not include handling and holding yards, hospital pens and other seldom-used pens. These other pens would be defined as a part of the non-vegetated surface within the feedlot and runoff would be estimated separately using algorithms outside the scope of this paper. AreaPen $\left(m^{2}\right)=$ StockingDensity $\times$ MaximumCapacity /No.Pens

The Market Type inputs (Figure 2) allow up to four different animal market types to be specified according to their entry and exit liveweights, daily liveweight gain and manure composition. The number of occupied pens for a particular market type $m$ is initialised (2), taking into account all the pens for a market type that are not occupied. The number of cattle per pen $p$ that is occupied is then initialised (3), converting Standard Cattle Units (SCU) to actual number of animals in according to the specified exit live weight of the market type assigned to that pen. (One SCU equates to a beast with an exit weight of $600 \mathrm{~kg}$.) The number of animals in each pen is summed to obtain the total head in the feedlot. Pens designated for market type $m$ will have animals at various stages of growth and so animal weights (LiveWeight $t_{\text {, day }=0} \mathrm{~kg} / \mathrm{head}$ ) are initialised to range from EntryWeight $_{m}$ to ExitWeight . $_{\text {. }}$

No.PensOccupied ${ }_{m}=$ No.Pens $\times$ ProportionOfTotalHead $_{m} / 100 \times$ ProportionOfPensOccupied $_{m} / 100$

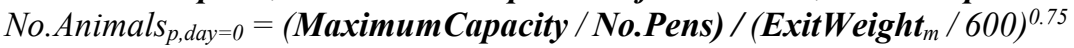

Pen Management inputs (Figure 2) allow the manure pad depths to be initialized, with each pen assigned a different depth of manure pad build-up ranging from PadDepthAboveBaseAfterCleaning to $2 \times$ PadDepthAboveBaseAfterCleaning, and a different number of days since cleaning in accordance with the degree of manure build-up indicated by the pad depth, up to a maximum number of MinDaysBetweenCleaningEvents, the minimum number of days between cleaning events. The initial masses of VS, TN, TP and Salts in each pen are assumed to be $40 \%, 2.5 \%, 0.7 \%, 3.0 \%$ (respectively) of the initial mass of manure (TS) in the pen. 
The Manure Pad Hydrology inputs (Figure 2) allow the mass of manure (total solids or TS) in each pen to be initialised (4). PadBulkDensity is the Pad bulk density $\left(\mathrm{kg} / \mathrm{m}^{3}\right)$ which is approximated by the bulk density of the predominant (lower) pad layer (BulkDensityLowerLayer or BulkDensity layer $=l o w e r_{\text {) }}$. The gravimetric water content of the manure pad is initialized to $60 \%(\mathrm{~g} / \mathrm{g})$ dry manure weight basis. The two layers of the manure pad have the initial manure mass apportioned between the upper and lower layers using a 20:80 ratio. Similarly, the mass of water (MassWater ${ }_{p}$ ) is apportioned assuming a 50:50 ratio. This allows the initial gravimetric moisture content of each pad layer to be calculated (5), with the moisture contents of both the upper and lower layers of the pad constrained to lie within the user-defined MinimumMoistureContent or PadMCMin (i.e. air dry moisture content) and MaximumMoistureContent or PadMCMax. The manure depth of each pad layer of a pen is then initialised (6).

MassTS $_{p, \text { day }=0}(\mathrm{~kg})=$ PadDepth $_{p, \text { day }=0} \times$ AreaPen $\times$ PadBulkDensity $\times 0.001$

$P_{\text {PadMC }} C_{\text {layer }, \text { day }=0}(\% \mathrm{~g} / \mathrm{g}$ dry weight basis $)=$ MassWater $_{\text {layer }, \text {, day }=0} /$ Mass TS $_{\text {layer }, p} \times 100$

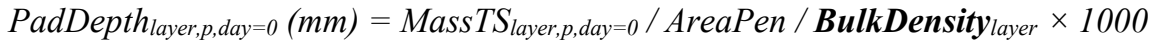

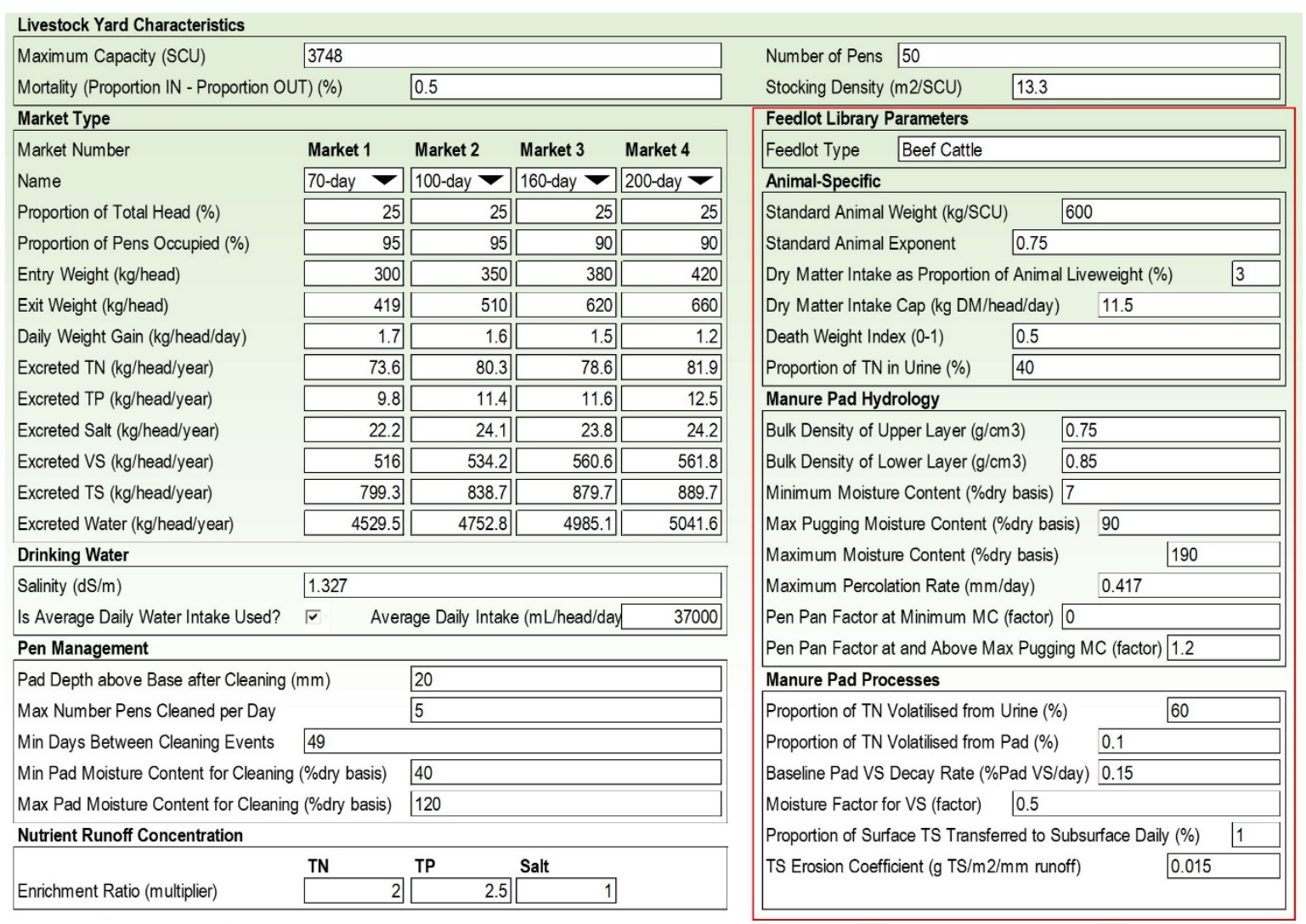

Figure 2. MEDLI screen layout for feedlot production pens showing the inputs required (with units and some example values). Default inputs for beef cattle (the Feedlot Library Parameter group box) will be supplied from the model library but these can be changed by the user when better information is available.

\subsection{Daily Calculations for each pen}

Following feedlot pen initialisation, daily calculations are performed on a pen by pen basis, to model the key processes of herd dynamics, manure production, assimilation of the fresh manure into the pad, pen hydrology and pen cleaning. The model determines the mass of water (MassWater ${ }_{p, d a y} k g$ ), and the mass TS, VS, TN, TP, and salts (denoted $X X)\left(\right.$ Mass $\left.X X_{p, d a y} \mathrm{~kg}\right)$ that are present in the manure pad in pen $p$ on day day following rainfall and excretion, volatilisation of $\mathrm{TN}$ or evaporation of water, runoff or erosion of solids, and pen cleaning using (7) and (8). Calculation of the mass terms are detailed in the Sections below.

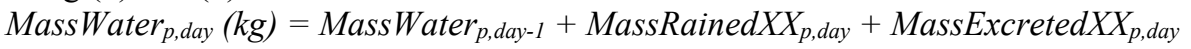

- MassEvaporation $X X_{p, \text { day }}$-MassRunoff $X X_{p, \text { day }}-$ MassCleaned $X X_{p, \text { day }}$

$\operatorname{Mass} X X_{p, \text { day }}(\mathrm{kg})=\operatorname{Mass} X X_{p, \text { day-1 }}+$ MassExcreted $X X_{p, \text { day }}$

$$
\text { - Mass Volatilised } X X_{p, \text { day }}-\text { MassEroded } X X_{p, \text { day }}-\text { MassCleaned } X X_{p, \text { day }}
$$




\section{Herd dynamics}

For each occupied pen, animal live weight $(\mathrm{kg})$ for each market type $m$ is increased according to the specified daily weight gain (9). When animals in a pen reach the exit weight for their market type, the model flags that the pen is vacant and drafts another mob (of the same market type) into another vacant pen if possible (the pen that has been vacant the longest) or else into the same pen. Mortality, defined as the percentage of animals entering the feedlot that die, is modelled by assuming that an animal is only vulnerable to death on the day they are at their "death weight" (kg), specified by the user via a DeathWeightIndex to lie between EntryWeight ${ }_{m}$ and ExitWeight $_{m}$. When animals in pen $p$ reach their death weight, the number of animals from that pen that die on that day is calculated as a function of the specified mortality, the cumulative number of animals that have obtained their death weight since the start of the simulation (CumNoVulerable), and the cumulative number of mortalities since the start of the simulation (CumNoMortalities) (10). The number of animals remaining in pen $p$ is then updated (11). Pens that have no live animals left will remain vacant until the next normal restocking day for that pen.

LiveWeight $_{p, \text { day }}(\mathrm{kg})=$ LiveWeight $_{p, \text { day }-1}+$ DailyWeightGain $_{m}$

No.Dead , day $($ head $)=\%$ Mortality $/ 100 \times$ CumNoVulnerable - CumNoMortalities

No.Animals day $($ head $)=$ No.Animals Aday-1) $_{\text {Ano.Dead }}$ day

\section{Manure production}

Manure is only generated within occupied pens. The mass of TN, TP, Salt, VS, TS and water (denoted $X X$ ) that is excreted each day and added to the manure pad is estimated (12). The user-specified annual mass excreted per head can be sourced using models such as BEEFBAL (QPIF 2004). Salt excretion only refers to the dietary salt excreted and any salt intake during drinking is accounted for using (13) and (14). Water intake may be derived from a user-defined constant AverageDailyIntake or calculated as a function of the dry matter intake and the day's average temperature $\left({ }^{\circ} \mathrm{C}\right)(15)$. Dry matter intake depends on the size of the animal, and is capped at a value of DryMatterIntakeCap (16).

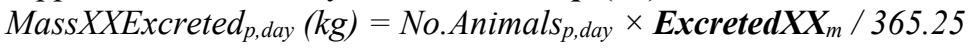

MassSaltExcreted $_{p, \text { day }}(\mathrm{kg})=$ No.Animals $_{p, \text { day }} \times$ ExcretedSalt $_{m} / 365.25+$ MassSaltDrank $_{p, \text { day }}$

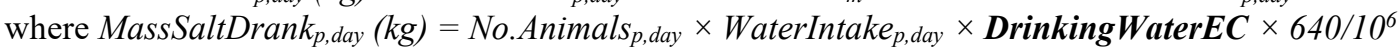

WaterIntake $_{p}(\mathrm{~kg} / \mathrm{head})=$ DryMatterIntake $_{p} \times\left(3.413+0.01592 \times e^{\left(0.17596 \times t_{\text {day }}\right)}\right)$

DryMatterIntake $_{p}(\mathrm{~kg}$ DM/head/day) $=$ MINIMUM (DryMatterIntakeCap,

LiveWeight $_{p, d a y} \times$ DryMatterIntakeAsProportionOfAnimalLiveweight)

\section{Assimilation of the fresh manure}

Firstly, the pad VS and TS (denoted $X X$ ) must be updated with the addition of excreted solids and the

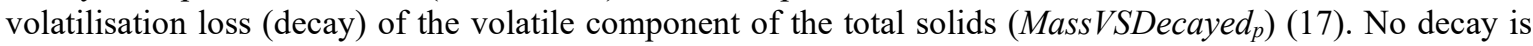
assumed to occur while the pad depth is at PadDepthAboveBaseAfterHarvesting, the specified depth remaining after pen cleaning. If pad depth exceeds this depth, the baseline daily decay of the VS component of the pad (BaseLinePadVSDecayRate or BDR) is adjusted according to pad surface temperature $(K t)$ and moisture content $(\mathrm{Km})$ to estimate MassVSDecayed $_{p \text {,day }}(18)$ and (19). The pad surface temperature is estimated from the AverageAirTemperature or $\boldsymbol{T}$ (20), while PadMC $C_{p, d a y}\left(\% \mathrm{~g} / g\right.$ dry weight basis) $=$ Mass Water $_{p, \text { day }} /$ $\operatorname{Mass}_{\text {T }}$,day $\times 100$ as per (5).

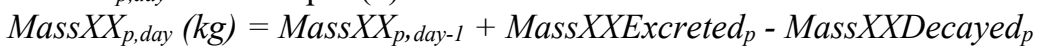

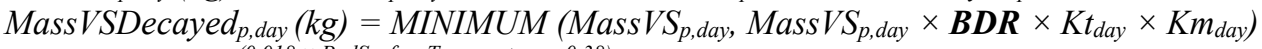

where $K t_{\text {day }}=10^{(0.018 \times \text { PadSurfaceTemperature }-0.38)}$ and where $K m_{\text {day }}=0.5 \times\left(1+\right.$ PadMC $_{\text {,day }} /$ PadMCmax $)$

PadSurfaceTemperature $_{\text {day }}\left({ }^{\circ} \mathrm{C}\right)=\left(5 \times \boldsymbol{T}_{\text {day }}+4 \times \boldsymbol{T}_{\text {day }-1}+3 \times \boldsymbol{T}_{\text {day }-2}+2 \times \boldsymbol{T}_{\text {day }-3}+\boldsymbol{T}_{\text {day- }-4}\right) / 15$

The mass of solids in the individual pad layers are also updated, with excreted TS added to the upper layer, and VS loss removed from both pad layers in proportion to their relative manure depths. As the daily calculations progress, any change in solids mass (or water mass) in the pad layers will impact on a number of pad attributes (layer depth, moisture content, the mass and depth of water present when the layer is at PadMCmax and PadMCmin) and so will always trigger a recalculation of these attributes. When the depth of the upper layer is more than one tenth that of the lower layer, a user-specified proportion of the total solids (ProportionSurfaceTSTransferredToSubsurfaceDaily or TSTransferred) of the upper layer is redistributed to the lower layer (21). This helps maintain a fairly constant manure depth on the surface as the lower layer depth increases while the pen remains uncleaned. Also, no more than $90 \%$ of the upper layer is removed during a cleaning event, with the balance coming from the lower layer. Effectively, this ensures there is always a surface (crust) layer present. The redistributed mass also contains water which must be transferred to the lower layer and this is done using the same transfer coefficient TSTransferred (22). However, the mass of water transferred must not cause the moisture content of the lower layer to exceed its maximum pad moisture content 
(PadMCmax) and so the amount of water transferred is limited to the water deficit of the lower layer with any "excess" water left in the upper layer.

MassTSRedistributed $_{p, \text { day }}=$ TSTransferred $\times$ Mass TS $_{\text {layer }=\text { upper }, \text {, day }}$

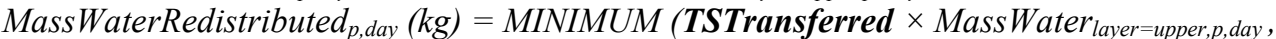

Mass WaterMax layer $=$ lower,, day $_{-}$Mass Water layer=lower,, ,day $)$

where (MassLowerLayerWaterMax) is calculated as per (5) (rearranged).

Secondly, the excreted water is added to the pad (23) and the moisture contents in the two pad layers are allowed to equilibrate, redistributing water from the wetter layer to the drier layer.

The potential depth of water redistributed by equilibration is determined as the minimum of the (i) maximum percolation rate across layers (MaximumPercolationRate), (ii) the current water deficit in the drier layer relative to the pad (average) moisture content and hence the maximum depth of water that the drier layer "demands", and (iii) the air dry limit of the wetter layer and hence the maximum depth of water that the wetter layer can "supply". The actual depth of water redistributed (DepthWaterRedistributed D,day $_{\text {) }}$ ) is calculated from the potential depth of water redistributed using a linear function based on the ratio of the wetter layer's moisture content over the drier layer's moisture content (24). This equation further constrains the depth (and hence mass) of water redistributed by equilibration such that the closer the ratio approaches one, the depth of water redistributed approaches zero.

Updated MassWater $_{\text {day }}(\mathrm{kg})=$ MassWater $_{p \text { day }}+$ MassWaterExcreted $_{p}$

DepthWaterRedistributed $_{p, \text { day }}(\mathrm{mm})=$ MINIMUM $_{\text {(PotentialDepthWaterRedistributed }}$,day,

$\left\{\right.$ PadWetterLayerMC $_{p, \text { day }} /$ PadDrierLayerMC $\left.C_{p, \text { day }}-1\right\} \times$ PotentialDepthWaterRedistributed $\left._{p, \text { day }}\right\}$

Lastly, the excreted TN, TP and Salt (denoted $X X$ ) are added to the pad (25). For TN, any ammonia-N volatilisation is also subtracted (26). The calculation for ammonia-N volatilisation losses includes an immediate loss component due to ammonia volatilisation from any fresh urine $\mathrm{N}(27)$ and a slower loss component from other nitrogen sources in the pad, assuming a volatilisation rate of $0.1 \% \mathrm{TN} /$ day from these other sources (28).

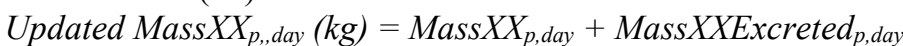

Updated MassTN $_{p \text { day }}(\mathrm{kg})=$ MassTN $_{p, \text { day }}+$ MassTNExcreted $_{p, \text { day }}$-ImmediateVolat Im day $_{\text {-OtherVolat }}$,day

ImmediateVolat $_{p, \text { day }}(\mathrm{kg})=$

ProportionOfTNinUrine $\times$ ProportionOfTNVolatilisedFromUrine $\times$ Mass TNExcreted $_{p \text { day }}$

OtherVolat $_{p, \text { day }}(\mathrm{kg})=0.001 \times$ MassTNExcreted $_{p \text { day }}$

\section{Pen hydrology}

Now that the manure has been assimilated, the pen hydrology is modelled to estimate any runoff on that day. If runoff is substantial, the pad may erode, reducing the pad depth. Vacant pens still contribute to runoff, and so the pad moisture content of all pens is estimated.

Firstly, any rain (mm) on the day is added to the upper layer of the pad.

Updated Mass Water $_{p \text { day }}(\mathrm{kg})=$ MassWater $_{p, \text { day }}+$ Rain $_{\text {day }} \times$ PenArea

Updated MassWaterlayer $=$ upper,, day $(\mathrm{kg})=$ MassWaterlayer $=$ upper,, ,day $+\boldsymbol{R a i n}_{\text {day }} \times$ PenArea

Secondly, pad evaporation is modelled. Well-managed feedlot pads display a propensity for rapid initial drying followed by a much slower phase once the surface starts to seal. The depth of "rapid" evaporation is removed from the upper layer of the pad (30), but this phase can only remove water down to its minimum (air dry) moisture content limit in the upper pad layer (PadUpperLayerDepthWaterMin), and according to the evaporative potential of the atmosphere on that day (31). The evaporative potential requires a "pen pan factor" which is calculated as a function of the pad moisture content (32) where $a$ is the intercept and $b$ is the slope of a linear relationship between PenPanFactor and pad moisture content defined by the user-specified data points (PadMCmin, PenPanFactorAtMinimumMC) and (PadMCMaxPug, PenPanFactorAt\&AboveMaxPuggingMC). After the pad upper layer has evaporated, water redistribution is modelled as per (24). The second "slower" evaporation phase is then modelled, removing any available water from the pad upper layer to satisfy any remaining evaporative potential of the atmosphere (33). The mass of water in the pad and the pad upper layer is updated for the loss of this slower evaporation component.

Updated Mass Water $_{p \text { day }}=$ Mass Water, $_{p \text {,day }}-$ DepthRapidEvap $_{p, \text { day }} \times$ PenArea

Updated MassUpperLayerWater ${ }_{p \text {,day }}=$ MassUpperLayerWater ${ }_{p, d a y}-$ DepthRapidEvap, $_{p \text {,day }} \times$ PenArea

DepthRapidEvap, day $_{(\mathrm{mm})}=$ MIN (PadUpperLayerDepthWater ${ }_{\text {,day }}-$ PadUpperLayerDepthWaterMin $_{p, \text { day }}$,

PanEvaporation day $\times$ PenPanFactor $)$

PenPanFactor $=(P a d M C-a) / b$

DepthSlowEvap $_{p, \text { day }}=$ MINIMUM (PadUpperLayerDepthWater, day - PadUpperLayerDepthWaterMin $_{p, \text { day }}$,

PanEvaporation $_{\text {day }} \times$ PenPanFactor - DepthRapidEvap, day $)$ 
Thirdly, the runoff from the pad is removed. Runoff will occur whenever the pad moisture content in any layer exceeds the layer's maximum moisture content (PadMCmax). Runoff depth is calculated for each layer (34) and then summed to determine the total depth of runoff, DepthRunoff $f_{\text {,day }}(\mathrm{mm})$. The mass of water remaining in the pad (Mass Water , day $_{\mathrm{kg}}$ ) is also updated.

DepthRunofflayer,p,day $(\mathrm{mm})=$ MAXIMUM( 0, DepthWater layer,p,day - DepthWaterMax $\left._{\text {layer,p,day }}\right)$

Fourthly, the eroded masses of TN, TP, Salts, VS, and TS from the pad (and also water associated with the eroded TS) (denoted $X X$ in (35)) are removed. For total solids, the mass eroded is predicted using (36), with a TS Erosion Coefficient or TSECoeff of about $0.015 \mathrm{~kg} \mathrm{TS} / \mathrm{m}^{2}$ per mm of runoff (Wise and Reddell 1973), and capped at $80 \%$ of initial mass TS in the pen. If the value for total solid erosion from the pen is less than $0.1 \mathrm{~kg}$, then no erosion of TS, VS, TN, TP and salts is assumed. Limited data suggests that the mass of volatile solids in runoff varies little and is around $50 \%$ of total solids for a range of feedlots and animal types. Hence, unless VS is excessive, the ratio of Mass VS $S_{p, d a y}$ to $\operatorname{Mass}_{T} S_{p, d a y}$ in the runoff is assumed to be 0.5 . This erosion estimate is capped to a maximum of $80 \%$ of the current VS in the pad (37). For TN, TP, and Salt (denoted $X X$ below in (38)), the mass of nutrient in the runoff is assumed to be proportional to the total solids in the runoff. A userdefined EnrichmentRatio $(\geq 1.0)$ for each nutrient is also included in the equation to take into account any additional nutrient entrainment as the runoff water flows over the pad surface. The EnrichmentRatio is best determined by calibrating predicted runoff concentrations to measured runoff or holding pond concentration data. Equation (38) is capped so that the amount eroded cannot exceed the total amount of nutrient present in the pad. With the reduction in total solids, the pad moisture content is recalculated. If the pad moisture content exceeds the maximum value (PadMCmax), it will be adjusted to PadMCmax, with any excess moisture added to the DepthRunoff $f_{\text {,day }}$. The mass of water in the upper and lower layers of the pad is then updated to account for this loss of excess moisture.

Updated Mass $X X_{p, \text { day }}(\mathrm{kg})=$ Mass $X X_{p, \text { day }}-$ MassEroded $X X_{p, \text { day }}$

MassErodedTS $_{p, \text { day }}(\mathrm{kg})=$ MINIMUM(TSECoeff $\times$ MassRunoff $f_{p \text { day }} \times$ PenArea, $0.8 \times$ MassTS $\left._{p, \text { day }}\right)$

MassErodedVS $S_{p, \text { day }}(\mathrm{kg})=\operatorname{MINIMUM}\left(\right.$ Ratio $\times$ MassErodedTS $\left._{p, \text { day }}, 0.8 \times{\left.\text { Mass } V S_{p, \text { day }}\right)}\right)$ where Ratio $=$ MAXIMUM $\left(0.5\right.$, Mass VS $_{p, \text { day }} /$ MassTS $\left._{p, \text { day }}\right)$

MassErodedXX $X_{p \text {,day }}(\mathrm{kg})=\operatorname{MINIMUM}\left(\right.$ Mass $X X_{p, \text { day }}$, Ratio $\times$ EnrichmentRatioXX $\times$ MassErodedTS $\left.S_{p, \text { day }}\right)$ where Ratio $=$ Mass $X X_{p, d a y} / \operatorname{Mass}_{p, \text { day }}$

\section{Pen Cleaning}

Pens are cleaned to remove the excess manure and in practice, involves scraping off excess manure without disturbing the impermeable layer. The cleaned masses of TN, TP, Salt, VS, TS and water (denoted $X X$ in (39)) is removed from the pad, assuming that the nutrient and salt composition of the pad is homogenous. Pen cleaning is triggered only when (i) the number of days since cleaning exceeds MinDaysBetweenCleaningEvents, and (ii) the pen's pad moisture content is within the range suitable for cleaning defined by MinPadMoistureContentForCleaning and MaxPadMoistureContentForCleaning and (iii) the pad depth is $20 \%$ greater than PadDepthAboveBaseAfterCleaning. Additionally, since there is a limit to how much manure can be cleaned daily, the total number of pens being cleaned in a day must not exceed a maximum number (MaxNo.PensCleanedPerDay). Once cleaning is triggered, the pad material above PadDepthAboveBaseAfterCleaning is removed, including solids, nutrients, salts and water. The amount removed from the pad is based on the proportion of pad depth removed (RemovedFraction ${ }_{p \text {,day }}$ ), calculated as (PadDepth $_{p, d a y}$ - PadDepthAboveBaseAfterCleaning)/ PadDepth ${ }_{p, d a y}$. For TS, the amount removed from the pad upper layer is capped at $90 \%$ of the layer TS, with the rest removed from the pad lower layer. This ensures that a minimum of $10 \%$ of the upper layer is reformed after cleaning, simulating compaction of the disturbed surface. The mass of TS removed from the pad (MassCleanedTS $\left.S_{p \text {,day }}\right)$ is the sum of the TS removed from each layer. For VS, TN, TP, and Salt (denoted $X X$ ), the amount removed is a simple proportion defined by the pad depth removed RemovedFraction ${ }_{p, \text { day }}$ (40). For water, the mass lost is associated with the cleaned TS (41). MassCleanedWater ${ }_{p, d a y}$ is the sum of the mass of water removed from each layer. The pad depth is then reset to PadDepthAboveBaseAfterCleaning, and the pad moisture contents are updated.

Updated Mass $X X_{p, \text { day }}(\mathrm{kg})=$ Mass $X X_{p, \text { day }}-$ MassCleaned $X X_{p, \text { day }}$

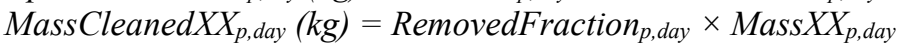

MassCleanedWater layer,, day $(\mathrm{kg})=$ MassCleanedTS $_{\text {layer, }, \text { day }} \times$ pre-cleaning PadMC layer,, day $\times 0.01$

\subsection{Runoff from all production pens}

The daily runoff volume is summed across all production pens in the feedlot to predict the volume of runoff for the day: VolumeRunoff day $\left(\mathrm{m}^{3}\right)=\Sigma\left(\right.$ MassRunoff $\left.f_{\text {,day }}\right) \times 0.001$

Runoff TN, TP and salt concentrations (denoted $X X)$ are calculated as:

ConcRunoff XX $_{\text {day }}(\mathrm{mg} / \mathrm{L})=\Sigma\left(\right.$ MassErodedXX $\left.X_{\text {, day }}\right) /$ VolumeRunoff day $_{\text {du }} \times 1000$ 


\subsection{Assumptions and Limitations}

We have attempted to model the complex dynamic processes within feedlot production pens that impact on the quantity and quality of runoff using a daily time step mass balance approach. A number of key processes are not well represented in this model, including the rate of pad evaporation after wetting and subsequent pugging of the pad, impact of rainfall intensity and duration and pen slope on nutrient entrainment in runoff, nitrogen volatilisation from feedlot pads, and salt dynamics in the feedlot system. Empirical relationships based on limited Australian data have been used to estimate the quantity of manure solids and nutrients eroded from the pad during a rain-day. The modelled pen cleaning may not reflect on-site practices such as the mounding of manure in small areas within the pens. In dry periods, pen cleaning may be managed by wetting the pad, a process not modelled. Given these limitations of the model, there remains a need to calibrate the model average runoff quality predictions to the chemistry of the holding pond receiving the runoff.

\section{VALIDATION}

Validation of the feedlot hydrological model was undertaken using the four field collected data sets from three South East Queensland feedlots and the same methodology as described in Atzeni et al. (2001). Results of statistical analysis conducted comparing feedlot pen rainfall runoff between in-field measured data and that predicted have indicated generally close correlation between the data sets (Figure 3). Data collection is in progress to allow more in-depth testing and validation of the feedlot module.

\section{CONCLUSIONS}

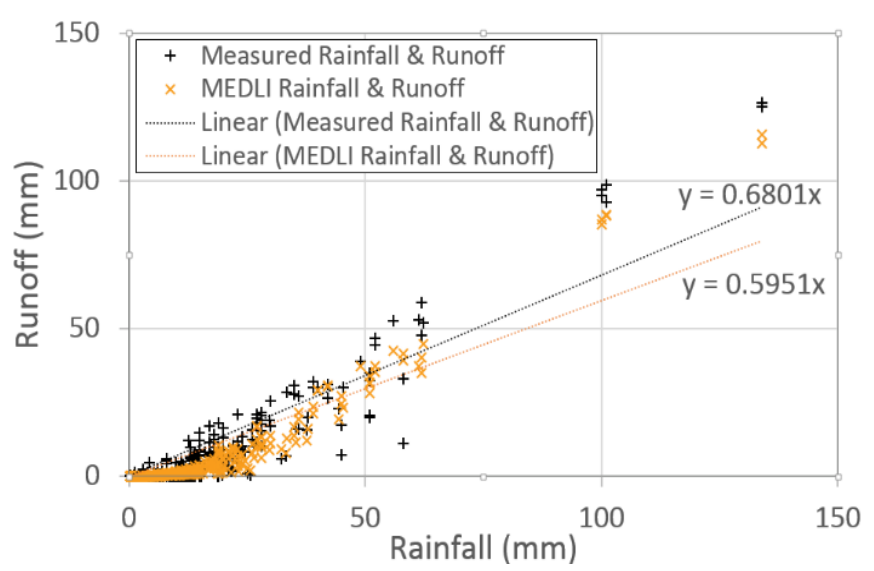

Figure 3. Measured runoff and MEDLI predicted runoff for all data sets.

The feedlot pen model allows MEDLI to estimate daily runoff from the feedlot production pens, along with the mass of manure solids, nutrients and salts carried by the runoff. This will enable the model to be useful as a design tool for designing effluent irrigation schemes for feedlots. Currently, the prediction of runoff quantity appears closely correlated with measured data. However, the runoff quality predictions require calibration of the TN, TP and salt the enrichment ratios with actual holding pond chemistry data from the site (if the modelling is for an expansion) or from a similar feedlot in the region (for a new application).

\section{ACKNOWLEDGEMENTS}

We gratefully acknowledge the funding of this project (B.FLT.5001) by the Meat \& Livestock Australia Limited (MLA), and the wonderful support provided by Jason Shen (Department of Environment and Science) and Margaret Jewell (Premise Pty. Ltd.).

\section{REFERENCES}

Atzeni, M, Casey, K., and A. Skerman (2001). A model to predict cattle feedlot runoff for effluent reuse applications. MODSIM 2001. In Proceedings of MODSIM 2001, vol. 4: General Systems, Canberra, pp. 1871-1876. https://www.mssanz.org.au/MODSIM01/Vol\%204/Atzeni.pdf

Atzeni, M., Watts, P., McGahan, E., and P. Nicholas (2015). Development of an odour emissions model for Australian feedlots. Part B. Modelling of feedlot hydrology using MEDLI. Final Report to Meat \& Livestock Australia. ISBN:9781741919752. https://www.mla.com.au/download/finalreports?itemId=3091

Gardner T., Vieritz A., Atzeni M., Beecham R., Littleboy M., Casey K., Sharma P., Farley T., Davis R., McGahan E., and P. Dillon (1996). MEDLI: A computer based design model for sustainable effluent disposal from intensive rural industries using land irrigation. In 'Land Applications of Wastes in Australia and New Zealand: Research \& Practice' (Eds. P.J. Polglase and W.M. Tunningley) pp 114-124. Proceedings $14^{\text {th }}$ Land Treatment Collective Meeting, Canberra, 29 September -4 October.

QPIF (2004). BEEFBAL - a nutrient mass balance model for beef cattle feedlots. Department of Employment, Economic Development and Innovation, Queensland Primary Industries and Fisheries. Version 10.01 obtainable through DAF Customer Service Centre, Department of Agriculture and Forestry, Queensland.

Wise, G.G. and D.S.L Reddell (1973). Water quality of storm runoff from a Texas beef feedlot, ASAE Paper No. 73-441, American Society of Agricultural Engineers, St Joseph, Michigan. 